\title{
PROYECCIONES FILOSÓFICAS DE ALGUNAS TEORIAS ETNOLINGÜISTICAS CONTEMPORANEAS
}

\author{
GERMÁN FERNÁNDEZ GUIZZETTI
}

(Universidad Nacional del Litoral - Universidad Católica de Santa Fe)

2a. PARTE

III

\section{EL RELATIVISMO LINGỨ́STICO}

El aspecto etnosicológico del relativismo cultural es el que incide directamente en el problema del conocimiento y de su veracidad. Aunque Herskovits apenas roza el problema en su último artículo ${ }^{57}$, ya anteriormente se había pronunciado por un relativismo epistémico de base lingǘstica: "Nos acercamos aún - escribe en 'El hombre y sus obras' - al problema de la naturaleza última de la realidad misma. Cassirer sostiene que la realidad solamente puede ser experimentada por medio del simbolismo del lenguaje. Entonces, la realidad, ¿no resulta definida y redefinida por los simbolismos siempre cambiantes de los innumerables lenguajes de la humanidad?" 58.

Por su parte, el trozo de la obra de Cassirer al que Herskovits se refiere, no puede ser más explícito en su negación de toda posibilidad de transcendencia ${ }^{59}$. En él, pareciera olvidar su autor que un símbolo lo es en cuanto conjunto de relaciones recíprocas entre significante y significado y que, por lo tanto, no puede, en modo alguno, ser reducido al mero significante, como se desprende del texto referido. El significante es sólo por, en cuanto y en referencia a su significado que lo trasciende en cuanto no se identifica con él.

Esta postura idealista parece empíricamente infundada. Las formas simbólicas son el medio de aproximarse progresivamente a la captación de "Lo Extramental". Es cierto que ellas constituyen el único medio de que dispone el hombre para la penetración de la realidad "en sí" que lo trasciende, pero esto no significa que deban ser consideradas ilusorias de lo cual se desprendería su inherente falacidad. Al contrario, el progresivo dominio que de lo cósmico han obtenido las ciencias físicas, demuestra 
que la "forma simbólica" dista mucho de ser una vía de escape a la realidad. Por el contrario, ella suple la natural indigencia de nuestras posibilidades epistémicas para penetrar y captar algo de la Realidad Extramental en cuanto tal.

Tampoco debe el símbolo ser interpretado como "el supuesto previo para todo lo que sea captar "objetos' o realidades" 60 de tal modo que lo real no pudiera concebirse "en sí" sino por referencia a lo simbólico. El signo, relación de significante a significado, adquiere su validez epistémica, no por ser un "a priori" previo a toda experiencia, sino por fundarse en las vivencias cotidianas de una realidad: el hombre que, aunque trasciende lo cósmico en cuanto actividad mental creadora ${ }^{61}$ es, por su naturaleza bio-física, parte del cosmos. De tal modo, el hombre se entronca en Lo Extramental pues él mismo es también y parcialmente algo de lo extramental: sus sentidos lo enlazan con lo extrasomático mediante procesos que obedecen a leyes cósmicas que escapan y trascienden la faz creadora del hombre-mente.

Pero, dejemos estos planteos filosóficos para las conclusiones y volvamos a nuestra actual tarea: la de mostrar como la postura relativista de fundamentación lingüística toma forma sistemática en la obra de

\section{Benjamin Lee Whorf.}

Este autor jamás llegó a publicar un libro en que sintetizara su pensamiento. Sin embargo, sus artículos constituyen, en sí, un cuerpo de doctrina bastante sólido y coherente. Ellos han sido recientemente reunidos y editados por John B. Carroll, bajo el título de "Language, Thought and Reality" ${ }^{2}$.

No es éste el lugar para llevar a cabo un análisis exhaustivo del pensamiento de Whorf en la multiplicidad de sus proyecciones. Me limitaré a exponer los aspectos que se refieren directamente al relativismo lingüístico y a sus proyecciones filosóficas. Estas últimas son estudiadas por nuestro autor en su monografía "Language, Mind and Reality", cuyas tesis habrán de ser analizadas al final del presente estudio.

Una idea fundamental es la que inspira implícita y, a veces, explícitamente, toda la concepción relativista de Benjamin Lee Whorf. Ella puede resumirse perfectamente utilizando la terminología filosófica del marxismo, que yo he elegido para exponerla en virtud de un evidente parentesco entre las falacias reduccionistas del tipo Hegel-Marx, por un lado, y la falacia lingüística de Whorf, por el otro. Desde un punto de vista epistemológico, lo falaz es exactamente lo mismo en uno y otro caso: el erigir un elemento o hecho cultural dado, en factor determinante de todos los demás.

La "falacia lingüísticista" considera las estructuras idiomáticas como base y el resto de los hechos sico-social-culturales, como meros epi- 
fenómenos de lo linguístico. Al ser el hecho-base algo naturalmente diversificado y al considerar el resto de las creaciones humanas como dependiendo de él, la conclusión relativista se impone como valedera; lo cual deja de suceder cuando se analizan las premisas y' se cuestiona fundadamente su validez en términos de una concepción humanista y totalista de lo idiomático en cuanto hecho cultural.

Esta supervaloración de lo lingüístico ya aparece en los primeros trabajos de Whorf. Así, afirma de la noción de significado que "se lo hallará íntimamente conectado con lo lingüístico; su principio es el simbolismo, pero el lenguaje (inglés language; resulta difícil saber si se refiere a la función simbólica o al idioma) es el gran simbolismo que da pie a todos los otros" 63 .

Pasemos ahora a analizar los diversos aspectos de la temática relativista de cuño lingüístico.

El primer punto que nos interesa analizar es el problema de los "universales lingüísticos" que este autor encara específicamente en sus monografías "Grammatical Categories", "Discussion of Hopi Linguistics", "Language; Plan and Conception of Arrangement" y "Gestalt Technique of Stem Composition in Shawnee".

El todo de la teoría whorfiana es bastante desigual. Quizás se deba ello a que jamás tuvo oportunidad de elaborar una obra de conjunto. Esta desigualdad aparece claramente si se compara su postura respecto al problema de los universales lingüísticos con la adoptada en temas específicamente metalingüísticos.

Nuestro autor dista mucho de negar la existencia de universales lingüísticos; lo prueba el mero hecho de haber elaborado un agudo esquema para la sistematización de los hechos idiomáticos ${ }^{64}$, cuya validez afirma para todo tiempo y lugar.

$\mathrm{Su}$ planteo consiste en negar que este problema pueda tener solución mediante la ilusión simplista de la gramática tradicional, concretada en la aceptación de las categorías propias de los idiomas indoeuropeos, muy especialmente, las de las llamadas lenguas clásicas ${ }^{65}$.

Tal actitud dista mucho del puro relativismo. Por el contrario, Whorf cree en la existencia de los universales linguísticos, pero sostiene la necesidad de establecerlos sobre bases más sólidas. Pero dicha necesidad sólo puede ser satisfecha por una vía estrictamente empírica. "Para hacerlo - nos dice - debemos reexaminar los tipos de categorías gramaticales que se encuentran en los idiomas, empleando una amplia visión universalista de los fenómenos lingüísticos, esbozando conceptos más o menos nuevos y efectuando los necesarios agregados a la terminología"66.

En realidad, vemos, al releer sus estudios de lingüística indoamericana, su "Some Verbal Categories in Hopi" y su "Gestalt Technique of Stem Composition in Shawnee" ${ }^{67}$, por ejemplo, como Whorf insiste en la 
determinación de las categorías ǵramaticales y de su valor semántico mediante una constante y sistemática referencia a lo que yo he denominado sentido endolingüísticó ${ }^{68}$. Esto es que toda determinación de unidadestipo debe extraerse de, y efectuarse según el sistema de relaciones recíprocas, peculiar e inherente a cada estructura a analizar.

Así, los universales lingüísticos resultan esquemas más amplios logrados a partir del estudio concreto de las estructuras individuales.

Veamos, pues, cuáles fenómenos lingüísticos pueden ser, según sus puntos de vista, considerados universales; propios de la naturaleza misma de lo idiomático.

Una exposición esquemática del problema nos la da Whorf en su "Language: Plan and Conception of Arrangement" 62 .

Lo que interesa destacar aquí, es que nuestro autor concibe la existencia de niveles o planos lingüísticos de índole universal; que tales planos no constituyen, en modo alguno, un sistema estático de casillas recíprocamente incomunicables, sino un sistema dinámico de partes interreferidas funcionalmente $y$, por lo tanto, susceptibles de condicionarse e influirse unas a otras ${ }^{69} ; \mathrm{y}$, finalmente, que trata las jerarquías lexémicas, como uno de los planos de la Morfosintáxis, que él denomina Semasiología, plano que coexiste junto a otros dos; el de la palabra y el de la oración. Esto último: el hecho de concebir el léxico a modo de inventario e incluirlo dentro de la gramática, forma parte de su concepción generativa del sistema idiomático, latente en lo que él denomina estructura encubierta. En efecto, el papel que Whorf atribuye a los fenómenos lexémicos dentro del funcionamiento de lo lingǘstico, es muy similar al que actualmente se le da en Gramática Transformacional.

En el artículo sobre las categorías gramaticales ${ }^{70}$, nuestro autor fundamenta el criterio que lo había movido a elaborar su "Plan" y, al mismo tiempo, establece la naturaleza de los universales lingüísticos que sirven de base a su esquema.

En primer lugar, es interesante destacar que el criterio whorfiano no da pie al distribucionalismo meramente "posicionalista", tan en boga en muchas descripciones de idiomas indoamericanos elaboradas en su país, y es preciso reconocerlo, en abierta contraposición con el pensamiento primero de la lingüística teorética estadounidense, concretado en la obra de Sapir.

Según Whorf, "este punto de vista (el "posicionalista") prescinde de la consideración de las varias clases de palabras marcadas, no por rótulos morfémicos, sino por y según tipos de esquematización, por ejemplo, por el evitar sistemáticamente ciertos morfemas; por la selección lexical; por el orden de las palabras, que también es orden de las clases; y, en general, según una configuración lingüística definida" ${ }^{71}$.

La principal distinción que él descubre, en el plano de los universales lingüísticos, es la existente entre categorías descubiertas (overt cate- 
gories) o fenotipos, y categorías encubiertas (covert categories) o criptotipos .

Las primeras se caracterizan por poseer una "marca formal presente... en toda oración que contenga un miembro de dicha categoría"72.

Las segundas, en cambio, "se hallan marcadas... solamente en ciertos tipos de oración (ya veremos como puede relacionarse esto con las modernas concepciones generativas de la gramática) y no en todas aquellas en que ocurra un elemento o palabra perteneciente a la categoría en cuestión. El ser dicha palabra miembro de una clase determinada, no resulta evidente hasta que se presenta el problema de usarla o de referirse a ella en uno de estos tipos específicos de oración, y, entonces, encontramos que pertenece a una clase la cual requiere cierto tratamiento distintivo, que puede ser también un tratamiento negativo consistente en la exclusión de aquel tipo específico de oración"73.

Esta característica de poder o no ocurrir en una dada secuencia típica con otros elementos propios de determinados tipos construccionales es lo que Whorf designa como la capacidad de reacción (reactance) de una categoría encubierta ${ }^{74}$.

Veamos qué importancia presentan estos aportes de Whorf para situar y valorar su obra dentro de la Lingüística General contemporánea.

Creo que en su noción de criptotipo, en cuanto funcionalmente opuesto al fenotipo, hay implícito una concepción dinámica y cuasigenerativa de la estructura idiomática. Ello convierte a Whorf, con las lógicas limitaciones impuestas por el grado de desarrollo de la Teoría Lingüística cuando escribiera sus estudios, en un precursor, aunque inconciente de serlo, de li. Gramática Transformacional contemporánea ${ }^{75}$.

Los criptotipos equivaldrían, en las actuales concepciones transformacionalistas, a miembros de clases formales caracterizadas por rechazar, por agramaticales, ciertas y determinadas operaciones transformacionales. En cuanto a lo que define al criptotipo; a esa propiedad de latencia estructural, es decir, de comportamiento funcional latente ${ }^{76}$, que Whorf designa como su "capacidad de reacción", tenemos que no se trata sino del grado de licitud o ilicitud, hoy denominado gramaticidad (grammaticalness), con que ciertas operaciones transformacionales pueden aplicarse a estructuras oracionales en las que figuran, como constituyentes, miembros de algunas clases formales. Así, los verbos intransitivos, que Whorf presenta como criptotipos a causa de su carencia de voz pasiva, serían actualmente definidos como miembros de una subclase de la clase formal "verbo"; subclase caracterizada por el hecho de que toda vez que alguno de sus miembros es constituyente de una frase verbal ${ }^{77}$, la oración a que pertenece dicha frase no puede ser transformada en pasiva, y, de serlo, la transformación (transform), u oración resultante de la anterior operación transformacional (trandformation), ha de ser necesariamente agramatical; ha de repugnar a los hábitos lingüísticos de los hablantes ${ }^{78}$. 
Simbólicamente ${ }^{79}$ tendremos:

$$
\begin{aligned}
& \text { Or. }= \text { Fr.N. }+ \text { Fr.V. = Prop. }+ \text { ivo. = Juan camina } \\
& \text { Fr.N. = Prop. = Juan } \\
& \text { Fr.V. = ivo. = camina }
\end{aligned}
$$

De donde:

$$
\text { Prop. }+ \text { ivo. } \longrightarrow \text { Prop. }+ \text { Fr.V.Pva. }
$$

Esto significa que la operación de sustracción y las dos operaciones de adición que, en español se requieren, en el constituyente frase verbal, para transformar en pasiva una creación nuclear (kernel sentence), no pueden efectuarse aqui porque el verbo caminar pertenece a la subclase funcional de los intransitivos; el resultado sería agramatical:

T. = Prop. + Fr.V.Pva. = Prop. + Aux. + R. ivo. + M. Part. = Juan es caminado.

Benjamin Lee Whorf llega, partiendo también de una concepción dinámica de lo idiomático, a las mismas conclusiones de la gramática transformacional: para algunas subclases de la clase "verbo", la operación transformacional "pasiva" es lícita y la transformación resultante, gramatical; para otra subclase, la de los verbos intransitivos, no lo es.

Finalmente, no puede dejarse de recalcar el hecho de que el postular la existencia de dos tipos de categorías funcionalmente opuestas y diferenciadas, muestra como el punto de vista whorfiano combina lo estático y lo dinámico, en su concepción del sistema. Todo idioma participa, pues, de ambos aspectos los cuales nos son dados por la universal coexistencia de categorías descubiertas y categorías encubiertas.

Esta naturaleza dual del idioma es concebida por nuestro autor como una coexistencia de factores diferenciados, pero también relacionados entre sí. La acabada comprensión del aspecto expreso de la estructura, el cual nos es dado por los fenotipos, requiere el conocimiento de su aspecto implícito y latente, concretado en el funcionamiento de los criptotipos que junto a ellos puedan coocurrir en la cadena hablada ${ }^{80}$. De este modo queda, pues, establecida la conexión funcional entre el aspecto estático y el dinámico de la gramática.

Con su concepción dualista del idioma y con su noción de criptotipo, Whorf anticipa, con sentido generativo, la solución de problemas que sólo la encontrarían, finalmente, en el actual estructuralismo transformacionalista, con su doble concepción de una gramática del núcleo (kernel grammar) y de otra que estudia las operaciones transformacionales y sus productos, las transformaciones; y que constituye la gramática transformacional (transform grammar).

Es el constituído por su distinción entre fenotipos yl criptotipos, el aspecto más importante de su aporte a la solución del problema de los universales lingüísticos. 
Podemos concluir que la postura whorfiana con referencia al problema de los universales lingüísticos, puede ser caracterizada, a pesar de más de una inconsecuencia que quizás hubiera desaparecido en una reelaboración de su pensamiento, como la de un mesurado pluralismo tipológicoestructural; pluralismo fundado en la diversidad funcional interna existente entre los idiomas-tipo, es decir, en el sentido endolingüístico típico de los fenómenos; cuyo sentido da fundamento a todo sistema.

Es interesante destacar aquí, algo que, aunque en el conjunto total de su obra quizás no pase de mera referencia, incide en el problema de la fundamentación metalingüística de los universales lingüísticos.

En su monografía "Gestalt Technique of Stem Composition in Shawnee", al analizar las contribuciones de la sicología de la forma para el estudio de la percepción visual, acentúa repetidamente el hecho de la unidad de naturaleza existente entre los hombres, a pesar de su diferenciación cultural y lingüística ${ }^{81}$. Cabe sin embargo preguntarse hasta qué punto este reconocer la importancia de la universal naturaleza sicobiológica del hombre y de su incidencia en la esquematización de la expériencia (de cuya incidencia no puede prescindir su simbolización pautada hecha sistema idiomático), puede compaginarse con el whorfiano principio lingǘstico de la relatividad, en la medida en que dicho principio considera 1.o idiomático como factor causante de la sistematización de nuestra experiencia no-lingüística, aún en el plano no-cultural de lo puramente síquico. En efecto, la actitud mesurada que Whorf muestra respecto a los universales, en el terreno de lo estrictamente lingǘstico, es lamentablemente abandonada cuando, como veremos más adelante, lo trasciende para llegar a lo metalingüístico. Allí, el hecho empírico de las diversidades estructurales lo lleva a extraer conclusiones apresuradas en el campo de la Etnosicología y' de la Sociología de la Ciencia.

\section{NOTAS}

57) MH 1958, pág. 267.

58) MH 1952, pág. 78.

59) Ernst Cassirer, "Antropología filosófica", versión española del original inglês por Eugênio Imaz, México, 1951; citado, de ahora en adelante, como EC 1945. Cfr. págs. 46 y 47.

60) Ernst Cassirer, "Las Ciencias de la Cultura", versión española del original alemán por Wenceslao Roces, México, 1951; citado, de aquí en adelante, como EC 1951. Cfr. pág. 51.

61) Germán Fernández Guizzetti, "Sicología, Lingüística y unidad de Lo Humano", comunicación al Primeiro Congresso Brasileiro e Jornada Latino-americana de Psicologia. Curitiba, diciembre 1953; citado, de aquí en adelante, como GFG 1953-b.

62) "Language, Thought and Reality. Selected Writings of Benjamin Lee Whorf". Cambridge-Massachussetts, 1956. 
Los trabajos estudiados en esta monografía, y a los cuales habré de referirme constantemente, son, además del ya citado en nota n. ${ }^{\circ}$ 44: "On the Connection of Ideas", citado como BLW 1929-a;

"On Psychology", citado como BLW 1927-b;

"The Punctual and Segmental Aspects in Hopi", citado como BLW 1936-a;

"A Linguistic Consideration of Thinking in Primitive Communities", citado como BLW 1936-b (fecha aproximada de redacción);

"Grammatical categories", citado como BLW 1945 (fecha de publicación);

"Discussion of Hopi Linguistics", citado como BLW 1937;

"Language; Plan and Conception of Arrangement", citado como BLW 1939-a;

"Some Verbal Categories of Hopi", citado como BLW 1938-b;

"The Relation of Habitual Thought and Behaviour to Language", citado como BLW 1941-a;

"Gestalt Technique of Stem Composition in Shawnee", citado como BLW 1940-a;

"Lingustic Factors in the Terminology of Hopi Architecture", citado como BLW 1953 ;

"Science and Linguistics", citado como BLW 1940-b;

"Linguistics as an Exact Science", citado como BLW 1940-c;

"Languages and Logic", citado como BLW 1941-b;

"Language, Mind and Reality", citado como BLW 1942.

63) BLW 1927-b, pág. 42.

64) BLW 1938-a.

65) Ibid., pág. 87.

66) Ibid.

67) BLW-b y 1940-a.

68) Germán Fernández Guizzetti, "Sentido, distribución y significado en el análisis funcional de las estructuras idiomáticas indoamericanas", publicado en el Vol. 6, N. 2 de la Revista de Antropologia, São Paulo, 1958 (citado de aquí en adelante como GFG 1958), pág. 193.

69) BLW 1938-a, pág. 132. Allí, al tratar los tipos derivacionales, sostiene expresamente su relación con los fenómenos morfológicos en términos que no dejan lugar a dudas acerca de su afirmación de las influencias recíprocas entre los planos idiomáticos: "Ellos (los tipos derivacionales) pueden fundirse o llegar a identificarse con las categorías morfológicas, y, en algunos idiomas, este apartado deberá transferirse del lexema, a la palabra: a la morfología".

70) BLW 1938-a.

71) BLW 1945, págs. 87-88.

72) Ibid., pág. 88.

73) Ibid., pág. 89.

74) Ibid.

75) Con referencia a la concepción generativa de la gramática, podemos dar la siguiente bibliografía fundamental: Noam Chomsky, "Syntactic Structures", La Haya, 1957.

Zellig S. Harris, "Co-occurrence and transformation in linguistic structures", publicado en el Vol. 33, N. ${ }^{\circ} 3$ de Language, Baltimore, 1957.

Fred W. Householder Jr., "On Linguistic Primes", publicado en el Vol. 15, N.o 2 de Word, New York, 1959.

Dean Stoddard Worth, "Transform Analysis of Russian Instrumental Constructions", publicado en el Vol. 14, N. os 2-3 de Word, New York, 1958. 
Sarah S. Gudshinsky, "Mazatec Kernel Constructions and Transformations", publicado en IJAL (International Journal of American Linguistics), Vol. 25, n. ${ }^{\circ}$ 2, Baltimore, 1959.

Robert B. Lees, "The Grammar of English Nominalizations", publicado como Vol. 26, N. 3 (parte segunda) de IJAL, Baltimore, 1960.

Las modernas concepciones generativas de la gramática, la llamada gramática transformacional, tienen por finalidad la de resolver problemas que no podían serlo por medio de un estructuralismo puramente distribucionalista. Así los que presentan las oraciones pasivas, en inglés o en español, las construcciones del tipo / nte s'e raīxu // en guaraní, y las neutralizaciones que se dan en las construcciones participiales y de infinitivo en griego clásico.

Prescindiendo de las diferencias existentes entre las diversas teorías transformacionalistas, todas ellas coinciden en distinguir dos planos fundamentales en la descripción de todo idioma: el de la gramática del núcleo (Kernel Grammar) y el de la gramática transformacional (Transform Grammar).

En la gramática del núcleo, y aunque muchos transformacionalistas sean más o rienos reacios en reconocerlo, se procede, fundamentalmente, en base a los métodos descriptivos estructurales (Sapir y, muy especialmente, Bloomfield) y según los planos descriptivos del estructuralismo pre-generativo. Según Chomsky, Kernel Grammar sería la que incluye un conjunto de oraciones elegidas "de tal manera que las secuencias terminales que subyacen al núcleo sean fácilmente derivadas a una descripción/E.F/ (se refiere al tipo descriptivo-estructural de naturaleza distribucionalista), mientras que toda ctra oración puede ser derivada de las antes mencionadas secuencias terminales, por medio de ciertas operaciones transformacionales simplemente establecidas". (Chomsky, op. cit., pág. 48).

En la gramática transformacional, se estudian los tipos de construcciones, transformaciones (transforms), derivados de las que constituyen el nível máximo de la gramática del núcleo, mediante operaciones transformacionales (transformations), las cuales exigen, como prerequisito, un exacto y exhaustivo análisis de las construcciones, o tipos construccionales, a transformar; análisis a efectuarse según los métodos estructurales tradicionales, es decir, lo que Chomsky denomina phrase structure.

Para Chomsky, "una operación transformacional $\mathrm{T}$ se efectúa sobre una determinada secuencia (o... sobre un conjunto de secuencias), cuya estructura posee determinados constituyentes, y la convierte en una secuencia con una estructura derivada que posee nuevos constituyentes" (Chomsky, op. cit., pág. 44).

Para Harris, "si dos o más construcciones (o secuencias de construcciones) que contengan las mismas $n$ clases (no importa qué contengan además) ocurren con los mismos n-tuplos de miembros de dicha clase en las mismas circunstancias oracionales (sentence environments)... decimos que las construcciones son transformaciones (transforms) las unas de las otras y que cada una de ellas puede ser derivada de cualquiera de las otras mediante una específica operación transformacional" (Harris, op. cit., póg. 288).

Finalmente, importa destacar cuál es, para los transformacionalistas, la finalidad de una descripción gramatical. Para Chomsky, el objetivo de una gramática se relaciona directamente con la predecibilidad de los hechos lingüísticos. Según él, una gramática no sólo ha de poder describir un cuerpo de enunciados ya registrados, sino también dar las pautas para la producción de un número de nuevos enunciados; enunciados que deberán resultar gramaticales. La gramaticidad (grammaticalness) de un enunciado se halla, para este autor, en relación directa su mayor o menor aceptabilidad 
por quienes hablan, como materno, el idioma a cuyos esquemas estructurales se pretende que responda el anunciado en cuestión. Así, tenemos que existen diversos grados de gramaticidad y agramaticidad, según un enunciado responda o nó, a las pautas vernáculas en cada uno de los diversos planos descriptivos de una estructura idiomática.

76) Esta latencia que yo califico de funcional y que es definitoria de la esencia misma del criptotipo, llevó a Whorf a comparar su noción lingüística de categoría encubierta, con la sicologica de inconciente. Así, sostiene que "la semejanza de los criptotipos con el concepto de inconciente freudiano y, más aún, quizás, con el junguiano, seguramente sorprenderá... aunque este paralelo quizás no deba ser llevado demasiado lejos". BLW 1937, pág. 111.

77) Por plano de la frase, entiendo el nivel estructural inmediato anterior al de la oración. Luego, las frases son los tramos (spans) en que una oración es dividida en una primera etapa analítica de segmentación.

78) GFG 1954, pág. 349.

"Los hábitos lingüísticos... (son los)... modos de simbolizar propios de una comunidad (noción sincrónica) que se concretan en las isoglosas cuyo conjunto nos da el idioma".

79) El simbolismo que empleo se funda en el que Noam Chomsky nos da en su "Syntactic Structures", del cual se aparta sólo en detalles. Doy, a continuación, las equivalencias correspondientes a cada abreviatura: Aux. (verbo auxiliar), Ava. (activa), Fr. (frase), ivo. (intransitivo), M. (marca), N (Sustantivo/nominal), Or. (oración nuclear), Part. (participio), Pl. (personal), Prop. (antropónimo), Pva. (pasiva) R. (raiz), T. (transformación), V. (verbo/verbal), + (adición) $=$ (reescripto como...), $\longrightarrow$ (operación transformacional ilícita).

80) BLW 1937, pág. 109.

"El significado de un fenotipo, aunque ostensiblemente claro, no puede ser plenamente comprendido en toda su sutileza, hasta que los criptotipos que van con él no hayan sido extraídos de su estado de subyacencia y sus significados vigentes, al menos en cierta medida, traidos a la conciencia".

81) BLW 1940-a, págs. 164/6. 